\title{
Adrenal neuroblastoma in an elderly adult: a case report and review of the literature
}

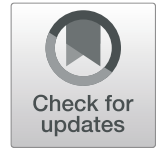

Haibin Zhang ${ }^{1}$ and Ziwei Feng ${ }^{2^{*}}$

\begin{abstract}
Background: Neuroblastoma is an embryonal malignancy of the autonomic nervous system and is the most common extracranial tumor of early childhood. However, neuroblastoma in adults is rare with an overall incidence of 1 in 10 million adults/year. Adults with neuroblastoma have a significantly worse prognosis than children with neuroblastoma.

Case presentation: In this case report we describe a 75-year-old Han Chinese woman with bilateral lower extremities weakness, numbness, and fatigue for 1 week. She initially presented as primary hyperaldosteronism with hypertension, persistent hypokalemia, and an elevated aldosterone/plasma renin activity ratio. An abdominal computed tomography scan with intravenously administered contrast showed a solid mass arising from her left adrenal gland. She underwent retroperitoneal laparoscopic surgery that allowed the resection of the mass. Microscopic and immunohistochemical staining, which were positive for synaptophysin, CD56, and vimentin, confirmed the diagnosis of adrenal neuroblastoma. Surgical resection of the tumor was done and no chemotherapy or radiation therapy was done postoperatively. She died from lung and brain metastases 22 months after surgical resection.

Conclusion: Adrenal neuroblastoma in elderly adults is a very rare disease with sparse data available in the literature. Early stage disease could be managed by surgical resection alone. However, the prognosis is significantly worse than that observed in pediatric patients. Further research focusing on tumor biology and therapy for this rare malignancy in adults may help to improve disease outcome.
\end{abstract}

Keywords: Neuroblastoma, Adrenal, Elderly, Adult

\section{Introduction}

A neuroblastoma (NB) is an embryonal malignancy of the autonomic nervous system and is the most common extracranial cancer of early childhood and the most frequently diagnosed malignancy in infancy. Common locations where NB can occur include the adrenal medulla (at least $50 \%)$, neck, chest, and pelvis $[1,2]$. The median age at diagnosis is 2 years, and more than $90 \%$ of patients are diagnosed under 10 years of age [3, 4]. The incidence of $\mathrm{NB}$ in adults is rare especially in the elderly population [5]. An adult with NB has a significantly worse prognosis than children, which may be due to different clinical course and response to therapy $[3,6]$. We discuss a rare case of an elderly patient who presented with lower extremities

\footnotetext{
* Correspondence: endourologic@126.com

2Division of Urology, Guangdong General Hospital, Guangdong Academy of Medical Sciences, No.106 Zhongshan Er Road, Guangzhou 510080,

Guangdong, China

Full list of author information is available at the end of the article
}

weakness and hypokalemia and was diagnosed as having adrenal NB.

\section{Case presentation}

A 75-year-old Han Chinese woman originally presented with bilateral lower extremities weakness, numbness with difficulty walking, and fatigue for 1 week. Her past medical history was unremarkable and she was healthy in general. She did not smoke tobacco or consume alcohol. She took no medication at home. She was married and had two children and both were healthy. There was no history of malignancies in her family. Her blood pressure was 147/100 $\mathrm{mmHg}$, heart rate was regular $82 /$ minute, and her body mass index (BMI) was $28.8 \mathrm{~kg} / \mathrm{m}^{2}$. A physical examination was normal. A laboratory workup disclosed decreased serum potassium level of $2.7 \mathrm{mEq} / \mathrm{L}$ and a normal serum sodium level of $135.9 \mathrm{mEq} / \mathrm{L}$. Her kidney function was within normal limits with blood urea nitrogen (BUN) of $8.9 \mathrm{mg} / \mathrm{dL}$ and a creatinine level of $0.79 \mathrm{mg} / \mathrm{dL}$. Further 
testing was notable for an elevated serum aldosterone level of $174 \mathrm{ng} / \mathrm{dL}$ (normal $\leq 21 \mathrm{ng} / \mathrm{dL}$ ), normal plasma renin activity (PRA) level of $7.59 \mathrm{ng} / \mathrm{mL}$ per hour, and elevated level of aldosterone/PRA of 22.9. Her 24-hour urine cortisol, vanillylmandelic acid (VMA), and metanephrine levels were unremarkable. She was initially diagnosed as having primary hyperaldosteronism and was given orally administered potassium and spironolactone. Muscle weakness and numbness were partially relieved. However, her serum potassium level was not corrected. Abdominal computed tomography (CT) scans with intravenously administered contrast showed a $4.5 \mathrm{~cm} \times 3.1 \mathrm{~cm}$ solid mass arising from her left adrenal gland (Fig. 1). Subsequently, she underwent retroperitoneal laparoscopic surgery that allowed the resection of the mass originating in her left adrenal gland. All the solid mass was excised, whereas her left adrenal gland was carefully dissected and spared. The mass was well circumscribed and its cut surface was grayish brown. It was surrounded by normal adrenal tissue. On macroscopic examination, the grayish brown mass measured $5.6 \times 4.5 \times$ $2.5 \mathrm{~cm}$. Microscopy revealed a tumor composed of small round blue cells forming rosettes with scant cytoplasm, fibrillary matrix material, hyperchromatic nuclei, and atypical mitoses (Fig. 2a). Immunohistochemical staining for CD99, epithelial membrane antigen, MyoD1, muscle actin monoclonal antibody (HHF35), chromogranin A, and S100 was negative with strong positive staining for synaptophysin (Fig. 2b-d), CD56, vimentin, and Ki67 (+ 30\%). On the basis of these findings, an adrenal NB was diagnosed. An I123-meta-iodobenzylguanidine (MIBG) scan was done postoperatively, which showed no hint of bone metastasis. No chemotherapy or radiation therapy was done based on our patient's decision. However, she died 22 months after resection due to bilateral lung and brain metastasis.

\section{Discussion}

NB is an embryonal tumor originating from the autonomic nervous system. The cell of origin is thought to be a developing and incompletely committed precursor cell derived from neural crest tissue. NB is the third most common childhood cancer [5]. However, the diagnosis is extremely rare in elderly patients with less than 100 cases reported in the literature. Our patient presented with NB in her adrenal gland associated with muscle weakness and persistent hypokalemia.

NB is rarely reported in the adult population, with $<10 \%$ of the cases diagnosed after age 10. The incidence rate for patients aged over 30 is 0.2 cases per million inhabitants per year and its incidence becomes increasingly scarce in the elderly population [5]. The clinical manifestation of NB in adult patients varies from asymptomatic to critically ill. It depends on the tumor's primary location and the extent of metastatic disease. The most common primary sites in an adult patient (18-60 years) are central nervous system (39\%) followed by retroperitoneum (17\%), while in elderly patients ( $>60$ years) the most common primary sites are soft tissue including heart (60\%) followed by trachea, mediastinum, and respiratory organs (31.4\%) [1]. Therefore, the most common presentations are abdominal mass, abdominal pain, muscle weakness, or presentations due to metastases such as back pain, bone marrow failure, spinal compression, and hypertension [4, 5, 7]. Besides muscle weakness, our patient also presented with hyperaldosteronism which is quite rare and is considered an

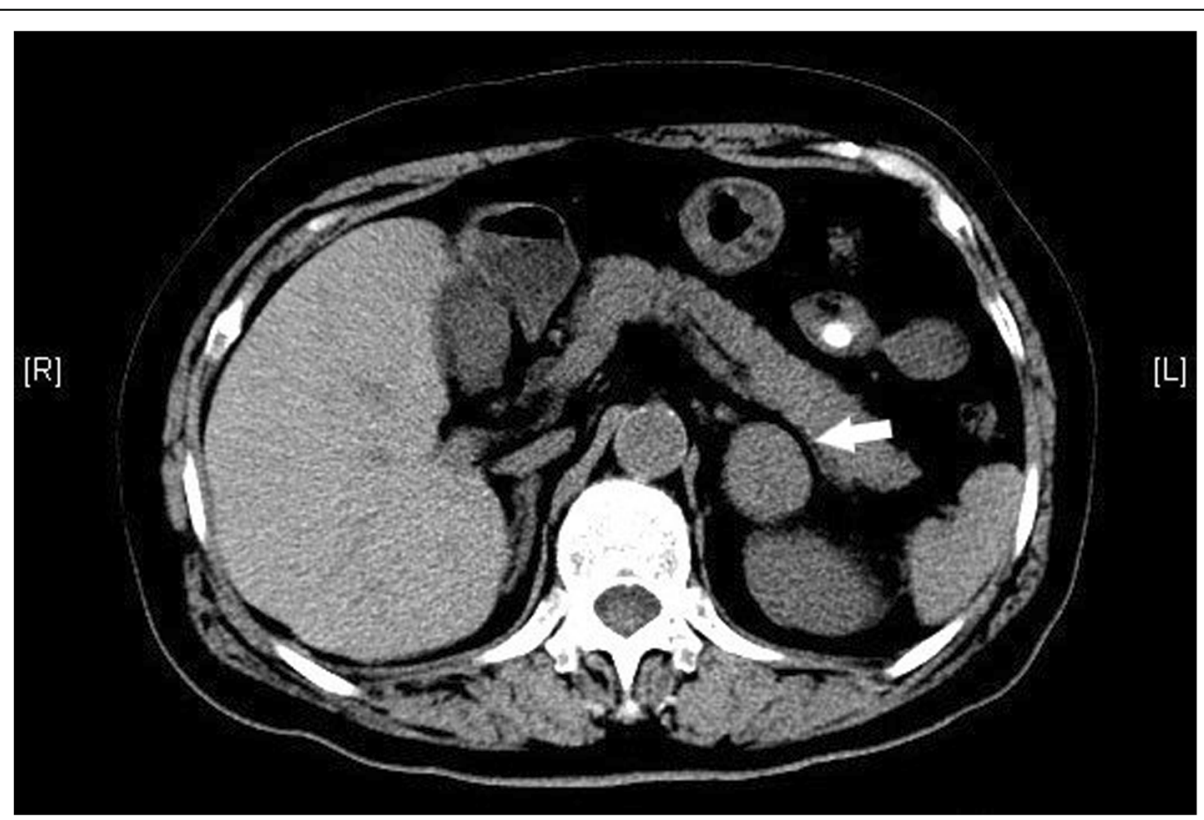

Fig. 1 Abdominal computed tomography image of a $4.5 \mathrm{~cm} \times 3.1 \mathrm{~cm}$ solid left adrenal mass (arrow) 


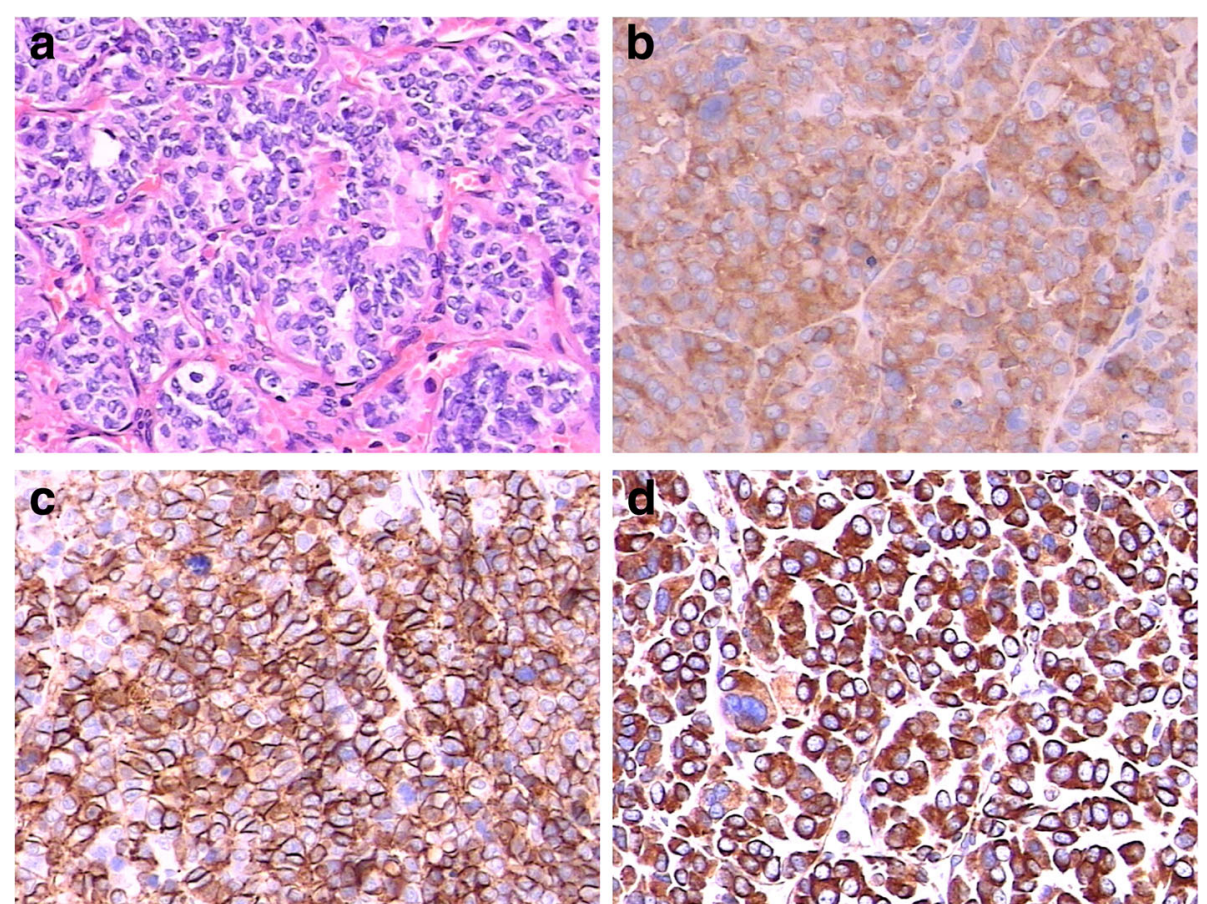

Fig. 2 Photomicrographs revealed various tumor properties. a Microscopy revealed a tumor composed of small round blue cells forming rosettes with scant cytoplasm, fibrillary matrix material, hyperchromatic nuclei, and atypical mitoses. The tumor stained positively for $\mathbf{b}$ synaptophysin, c CD56, and $\mathbf{d}$ vimentin

unexpected atypical presentation according to the medical literature [8]. The hyperaldosteronism can be explained by increased renin secretion secondary to the mass effect of the tumor compressing the renal artery [9].

A new International Neuroblastoma Risk Group (INRG) tumor staging system has been developed for the stratification of statistical and clinical risks of different subgroups of patients with NB. In the INRG Staging System, the extent of locoregional disease is determined by the absence or presence of image-defined risk factors (L1 and L2, respectively). Stage $M$ is used for widely disseminated disease [10]. Four broad categories of very low risk, low risk, intermediate risk, and high risk were proposed in terms of 5-year event-free survival with rates of $>85 \%,>75$ to $\leq 85 \%,>50$ to $\leq 75 \%$, and $<50 \%$, respectively. The category is based on age at diagnosis, INRG tumor stage, histologic category, grade of tumor differentiation, DNA ploidy, and copy number status at the $M Y C N$ oncogene locus and at chromosome 11q [2]. Our patient was classified as stage L2 of the INRG Staging System [10].

In our case, regarding treatment, we only performed surgical resection of the tumor but no chemotherapy or radiation therapy was done after the operation. The treatment strategy in pediatric patients with NB has been well studied; it includes surgical resection, and optimal combination chemotherapy and radiotherapy. However, there are no standard treatment guidelines or chemotherapy protocols for adult or elderly patients with NB due to the rarity of NB in this population [5]. A cohort study yielded 118 adult patients with NB from University of Texas MD Anderson Cancer Center; it concluded that for adult patients with L1 and L2 disease, a combination of surgical resection and radiotherapy offered better progression-free survival as well as overall survival than surgical resection alone. The median progression-free survival in patients with L1 and L2 disease was 11.1 months and 5.9 months, respectively. Chemotherapy did not show any additional benefit in outcomes among patients with L1 and L2 disease. The most common chemotherapy regimens employed in adult patients with $M$ stage disease were: cisplatin and etoposide alternating with carboplatin, vincristine, and cyclophosphamide (29\%); and vincristine and cyclophosphamide alternating with cisplatin, doxorubicin, and dacarbazine (24\%). The median overall survival of patients with M stage disease was 1.6 years [7]. Podda et al. conducted a series with 27 patients with NB aged 12-69 years in Italy [4]. The treatment protocol and outcome was as follows. Surgery only in stage I and surgery followed by radiotherapy in stage II. The 5 -year overall survival rate was $83 \%$ for stage I and II disease. Chemotherapy consisted of six cycles of cisplatin and etoposide alternating with Adriamycin (doxorubicin), cyclophosphamide, and vincristine and local therapy after sixth course consisting either of radiotherapy or surgery in stage III. In stage IV, ifosfamide was added to 
the cycles applied in stage III, followed by a consolidation phase with 10 Gy fractionated hemibody irradiation (HBI) or autologous stem cell rescue, and local therapy with surgery or radiation was scheduled after the fourth cycle. The 5 -year overall survival rate for patients with stage III and IV disease was $28 \%$ but all patients with stage IV disease relapsed and died due to disease progression [4].

\section{Conclusion}

NB in elderly adults is a very rare disease with sparse data available in the literature regarding natural history, genetic causes, treatment, and outcomes. Early stage adrenal NB in elderly patients can be managed with surgical resection alone. However, elderly adult patients have a worse prognosis than is observed in pediatric patients. Here we reported a rare case of an elderly patient with adrenal NB treated with surgical resection and with a survival of 22 months. According to the medical literature, surgical resection combined with local radiation therapy offers better outcomes in cases of adult local NB. Chemotherapy should be considered in disseminated disease. Further research focusing on tumor biology and therapy for this rare malignancy in adults may help to improve disease outcome.

\section{Abbreviations}

BMI: Body mass index; BUN: Blood urea nitrogen; CT: Computed tomography; HBI: Fractionated hemibody irradiation; INRG: International Neuroblastoma Risk Group; MIBG: Meta-iodobenzylguanidine; NB: Neuroblastoma; PRA: Plasma renin activity; VMA: Vanillylmandelic acid

\section{Acknowledgements}

Not applicable.

\section{Authors' contributions}

$\mathrm{HZ}$ : Drafted the manuscript and collected the patient data. ZF: Participated in the treatment of the patient and collected the patient data and critically revised it. Both authors read and approved the final manuscript.

\section{Funding}

Not applicable.

Availability of data and materials

Not applicable.

Ethics approval and consent to participate

Not applicable.

\section{Consent for publication}

Written informed consent was obtained from the patient's next-of-kin for publication of this case report and any accompanying images. A copy of the written consent is available for review by the Editor-in-Chief of this journal.

\section{Competing interests}

The authors declare that they have no competing interests.

\section{Author details}

'Zhongshan School of Medicine, Sun Yat-sen University, Guangzhou, China. ${ }^{2}$ Division of Urology, Guangdong General Hospital, Guangdong Academy of Medical Sciences, No.106 Zhongshan Er Road, Guangzhou 510080,

Guangdong, China.
Received: 16 April 2018 Accepted: 23 July 2019

Published online: 10 September 2019

References

1. Maris JM, Hogarty MD, Bagatell R, Cohn SL. Neuroblastoma. Lancet. 2007; 369(9579):2106-20.

2. Maris JM. Recent advances in neuroblastoma. N Engl J Med. 2010;362(23): 2202-11.

3. Kushner BH, Kramer K, LaQuaglia MP, Modak S, Cheung NK. Neuroblastoma in adolescents and adults: the Memorial Sloan-Kettering experience. Med Pediatr Oncol. 2003:41(6):508-15.

4. Podda MG, Luksch R, Polastri D, Gandola L, Piva L, Collini P, et al. Neuroblastoma in patients over 12 years old: a 20-year experience at the Istituto Nazionale Tumori of Milan. Tumori. 2010;96(5):684-9.

5. Rogowitz E, Babiker HM, Kanaan M, Millius RA, Ringenberg QS, Bishop M. Neuroblastoma of the elderly, an oncologist's nightmare: case presentation, literature review and SEER database analysis. Exp Hematol Oncol. 2014;3:20.

6. Esiashvili N, Goodman M, Ward K, Marcus RB Jr, Johnstone PA. Neuroblastoma in adults: Incidence and survival analysis based on SEER data. Pediatr Blood Cancer. 2007:49(1):41-6.

7. Conter HJ, Gopalakrishnan V, Ravi V, Ater JL, Patel S, Araujo DM. Adult versus Pediatric Neuroblastoma: The M.D. Anderson Cancer Center Experience. Sarcoma. 2014;2014:375151.

8. Gulleroglu K, et al. Neuroblastoma accompanied by hyperaldosteronism. J Renal Inj Prev. 2014;3(3):79-82.

9. Madre $\mathrm{C}$, et al. Hypertension in childhood cancer: a frequent complication of certain tumor sites. J Pediatr Hematol Oncol. 2006;28(10):659-64.

10. Cohn SL, Pearson AD, London WB, Monclair T, Ambros PF, Brodeur GM, et al. The International Neuroblastoma Risk Group (INRG) classification system: an INRG Task Force report. J Clin Oncol. 2009;27(2):289-97.

\section{Publisher's Note}

Springer Nature remains neutral with regard to jurisdictional claims in published maps and institutional affiliations.
Ready to submit your research? Choose BMC and benefit from:
- fast, convenient online submission
- thorough peer review by experienced researchers in your field
- rapid publication on acceptance
- support for research data, including large and complex data types
- gold Open Access which fosters wider collaboration and increased citations
- maximum visibility for your research: over $100 \mathrm{M}$ website views per year
At $\mathrm{BMC}$, research is always in progress.
Learn more biomedcentral.com/submissions 